\title{
XVI.
}

\section{Ueber die Beziehungen der sogenannten Steissdrüse zu den Steisstumoren.}

\author{
Von Dr. Martin B. Schmidt, \\ Assistenten am pathologischen Institut au Heidelberg.
}

Die Vermuthung eines Zusammenhanges zwischen einem Theil der angebornen Geschwülste der Steissgegend und der Steissdrüse stammt von dem Entdecker der letzteren, Luschka'), her, also ans einer Zeit, wo man in diesem Organ ein drüsenartiges Gebilde, ein Conglomerat von epithelerfüllten Blasen and Schläuchen erblickte. 2 Jahre später von Braune ${ }^{2}$ ) befürwortet und dahin präcisirt, dass sowohl „eigentliche Steissbeingeschwülste", also in der Hauptsache Sarcome, besonders "Cystosarcome", als auch "Sacralhygrome" aus jener hervorgehen sollten, hat sie sich bis zum heutigen Tage fortgeerbt und findet noch in den meisten Mittheilungen der neuesten Zeit über Sacraltumoren einen Wiederhall, olne von der fortschreitenden Erkenntniss über die wahre Structur der Steissdrüse beeinflusst worden zu sein. Arnold's ${ }^{3}$ ) Untersuchungen über die histologische Zusammensetzung der letzteren berichtigten Luschka's Deutung dahin, dass jene epithelartigen Zellen nur eine wandständige Auskleidung der Hohlräume bilden, welche letatere mit dem Lumen der Art. sacralis media in directem Zusammenhang stehen. Das ganze Organ stellt danach nur eine Gruppe von länglichen und runden Gefässerweiterungen dar, welche aus der genannten Arterie und ihren $Z$ weigen hervorgehen und deren Wand eine in allen Schichten stärker entwickelte Arterienhaut

1) Luschka, Die Steissdrüse des Menschen. Dieses Archiv Bd. XVIII. 1860.

2) W. Braune, Die Doppelbildungen und angebornen Geschwälste der Kreuzbeingegend. Leipzig 1862.

3) J. Arnold, Bin Beitrag zu der Struclur der sogenannten Steissrlüse. Dieses Archiv Bd. XXXII. 1865. 
bildet. Auf Grund dieser Befunde spricht Arnold der Steissdrüse die Fähigkeit ab, Geschwülste zu produciren, unter gleichreitigem Hinweis auf das nicht selten beobachtete Vorkommen histologisch gleichartiger Neubildungen im Zusammenhang mit den Meningen, wo eine Theilnahme der Steissdrüse undenkbar sei. Obwohl durch diese Untersuchungen Luschka's Lehre, wenn nicht ihre Erledigung, so doch eine Einschränkung hätte finden sollen, ist jene auch weiterhin von den Meisten, welche über Fälle der in Rede, stehenden Erkrankung berichten, in vollem Umfange gläubig angenommen worden. Sertoli ${ }^{1}$ ) stellt sich sogar, entgegen Arnold's Ansicht, im Anschluss an eigene histologische Untersuchungen als Fürsprecher derselben hin; Lachaud ${ }^{2}$ ) berichtet, dass Périn nach seinen Forschungen in der Steissdrüse eine Schweissdrüse erblicke, und ihre Fähigkeit zur Erzeugung der Tumoren anerkenne. Nur Wenige begegnen Luschka's Behauptung zweifelhaft, nur vereinzelte Stimmen haben sich im Anschluss an Arnold gegen sie erhoben. Sut$\operatorname{ton}^{3}$ ) weist ausser auf die vollständige Verschiedenheit der Steissdrüse und der Geschwülste in ihren elementaren Bestandtheilen vor Allem auch darauf hin, dass man in Abkömmlingen jener einen grossen Blutgefässreichthum erwarten müsse, der den auf sie bezogenen Bildungen grade in auffallend geringem Maasse eigen sei.

Wenn im Folgenden die grosse Zahl der Mittheilungen äber Einzelfälle von Sacraltumoren um eine neue vermehrt wird, so mag dies seine Berechtigung darin finden, dass dieselbe im Stande ist, den an sich zwar schon beweiskräftigen, aber doch nur aus Ueberlegungen hergeleiteten Gründen gegen die Lehre Luschka's eine anatomische Stätze zu verleihen.

Das Präparat, welches der Sammlung des hiesigen pathologischen Instituts entstammt, ist seit längerer Zeit in Spiritus aufbewahrt gewesen. Es

1) Sertoli, Ueber die Structur der Steissdrüse des Menschen. Dieses Archiv Bd. XLII. 1868 .

2) Lachaud, Recherches sur les tumeurs congénitales de la region sacrococcygienne. Thèse de Paris 1883.

3) J. Bland Sutton, On the origin of certain eysts ovarian, vaginal, sacral, lingual and tracheal. Journal of Anatomy and Physiology. April 1886. Vol. XX. Part III. 


\section{4}

stelit einen 8monatlichen Eotus weiblichen Geschlechts dar, welcher abgesehen von der ihm anhaftenden Geschwulst durchaus wohlgebildet erscheint: Der Kopfumfang entspricht der Grösse des Kindes; die Wirbelsäule zeigt keine Verkrümmung; alle Extrenitäten sind beä̈glich des Längen- und Dickenwachsthums, sowie der Stellung ihrer einzelnen Abschnitte frei ron Störungen. Die Cieschwulst selbst ist, offenbar während der Geburt, zum Theil von der hinteren Körperoberfläche ibres Trägers abgerissen, lässt sich jedoch leicht wieder in ihre ursprüngliche Lage bringen und mag zunächst in dieser beschrieben werden: Ihre ungefähr kreisrunde Basis nimont die Gegend des Kreuz- und Steissbeins und des hinteren Dammabschnittes ein und zieht nach rechts zu das dem M. glutaeus magnus entsprechende Gebiet vollstäudig in ibr Bereich, während linkerseits der äussere Theil desselben frei bleibt. Auf dieser Fläche erhebt sich die Geschwulst als ein zweifaustgrosser Körper mit einer Ausdehnung von $12 \mathrm{~cm}$ in der Länge und $9 \mathrm{~cm}$ in der Breite und einem $36 \mathrm{~cm}$ messenden Umfang. Nach unten zu wölbt sie sich über das Niveau des Dammes vor und reicht mit ihrem unteren Pol bis in die Höhe der Kniekeblen. Eine mechanische Beeinträchtigung ibrer Lage haben nur After und Geschlechtstheile erfahren: Der erstere ist nach rechts und vorn gedrängt und liegt au der Vorderfläche des Tumors; die Schamspalte weicht mit ibrem hinteren Ende nach rechts von der Mittellinie ab. Die Haut des kindlichen Körpers geht continuirlich auf die Geschwulst über und äberzieht sie in ganzer Ausdebnung; an der Basis von noch unverändertem Ausseben verdünnt sie sich allwählich und stellt auf der Höhe nur eine dünne, von platten Venen durchzogene Nembran dar, welche die Geschwulstmasse weiss. lich-gelb durchscheinen lässt. Die Oberfläche trägt einzelne durch seichte Furchen von einander geschiedene flache Wölbungen, ist aber im Uebrigen glatt.

Schlägt man nun den von oben ber von seiner Unterlage abgerissenen Tumor nach unten zurück, so siebt man, dass er nur noch durch einen Theil seines Hautüberzuges mit dem Körper des Kindes in Zusammenbang steht. Die ihm zugehörige Rissebene zeigt mehrere flach gewölbte Prominenzen, deren Fläche, abgesehen von einzelnen aufliegenden membranösen Fetzen von Bindegewebe glatt ist; drängt man diese Vorwölbungen auseinander, so erkennt man in ibuen $A$ bschnitte von ungefäbr wallnussgrossen kugligen und eiförmigen, an einander sich abplattenden Gebilden von weicher Consistenz, welche durch lockeres Bindegewebe zusammengehalten werden. Am kindlichen Körper wird durch das Zurückschlagen der Geschwulst der Beckenausgang freigelegt; derselbe ist zu einer fast kreisrunden thalergrossen Oeffnung dadurch umgebildet, dass das Kreuzbein sich nach hinten umgebogen hat und die von ihm nach dem Sitzbein ziehenden Bänder geschwunden sind; in der oberen Hälfte des Umfanges wird er umgeben von einer durchschnittich $3 \mathrm{~cm}$ hohen Hautmanschette, aus welcher sicb der basale Theil des Tumors beim Abreissen ausgeschält hat. Das $1 \mathrm{~cm}$ lange Steissbein liegt in dieselbe eingebettet; mit ibrer Innenfläche hängen beiderseits die untersten nach hinten vorgestülpten Abschnitte der Mm. glutaei magni, 
soreit sie gewöhnlich den Beckenausgang decken, zusammen. In der beschriebenen Oeffnung nun wird eine Masse sichtbar, welche im Aussehen voliständig der der äusseren Geschwulst gleicht und offenbar einem in die Leibestoohle des Kindes vordringenden Fortsatz derselben angehört; auch auf ihrer Rissfläche treten flachgewölbte Segmente von weichen kugligen, durch loses Bindegewebe aneinander gehaltenen Körpern hervor. Mastdarm und Vagina mit dem zusammengedrückten M. levator ani liegen der hinteren Fläche der Symphyse an. Von der eröfneten Bauchhöhle aus gesehen erweckt der intraabdominelle Theil des Tumors beim ersten Blick den Eindruck des schwangeren Uterus: der Fundus des symmetrisch gelagerten birnförmigen Körpers reicht bis zum Theilungswinkel der Aorta in der Höhe des 2. Lendenwirbels; seine Hinterfläche liegt der Wirbelsäule dicht an, während die vorderen und die beiden sejtichen Flächen einen Peritonäaläberzug besitzen, in dessen Falten der Nastdarm und die Ureteren verlaufen. Schält man, was sich leicht ausführen lässt, den Zapfen aus seiner Banchfellbekleidung aus, so erscheint or zunächst von einer dünnen Bindegewebskapsel umschlossen, welche, von ihrer Umgebung leicht trenribar, auch der Geschwulstoberfäche nur lose anhaftet nnd eine weitere bindegewebige Hülle deckt, von welcher aus, ebenso wie am äusseren Geschwulstabschnitt die lockereı Septen zwischen die kugligen Körper eindringen. Die Harnblase liegt plattgedrückt und mit dem Vertex nach rechts verschoben den Bauchdecken an; der Uterus ist ebenfalls comprimirt und nach der rechten Seite abgewicben, so dass das linke Lig. latum gegen das rechte bedeutend verlängert erscheint. Das Darmlabyrinth ist durch den Turoor von unten nach oben zusammengedrängt, jedoch bis auf eine Erweiterung der Flexura sigmoidea vollständig unverändert wie auch die übrigen Bauchorgane und Herz und Lungen ein durchans normales Verhalten aufweisen. Bei weiterer Präparation zeigen sich auch die Wirbelsäule, das Rückenmark und seine Häute und der Schädel mit seinem Inhalt durchaus regelmässig entwickelt. Der Hiatus sacralis ist geschlossen.

In seiner Gesammtheit stellt somit der Tumor einen sandubrförmigen Körper dar, dessen oberer kleinerer Abschnitt in die Leibeshöble des Kindes zwischen Wirbelsäule und Mastdarm eindringt, während der grösșere ausserhalb des kindlichen Körpers herabhängt; der beide verbindende Istbmus liegt in der Fbene des Beckenausgangs.

Eine besondere Aufmerksamkeit wendete ich der Aufsuchung der Steissdrüse zu. Die A. sacralis media, welche ich zu diesem Zwecke verfolgte, verläuft an der Hinterfläche der Bindegewebskapsel des intraabdominellen Geschwulsttheils, zwisehen ibr und der Wirbelsäule in der Medianlinie; ihre Präparation wurde elleichtert \{urch ihr abnormes Kaliber, welches ungefähr doppelt so stark ist, als man es bei neugebornen Kindern findet; makroskopisch wahmehmbare Aestchen dringen in den oberen Zapfen des Tumors nicht ein. An der Krämmungsstelle des Kreuzbeins, also in der dem kindlichen Körper angehörigen Rissfläche, zeigte sich das Gefäss durchtrennt, ohne dass das peripherische Ende zu entdecken war; um trotz des Mangels 
dieses Leitungsweges den Versuch, die Steissdrüse zu finden, nicht aufzugeben, schnitt ich das Steissbein, auf dessen Vorderfäche mehrere kleine, im gehärteten Zustand nicht zu bestimmende Gewebskügelchen sassen, mit seiner Umgebung aus und bettete es für die mikroskopische Untersuchung in Celloidin ein. In den von diesem Präparate serienweise angefertigten Längsschnitten, und zwar in den dem mittleren Theile des Steissbeins entsprechenden, findet sich dicht vor der Spitze desselben ein Gebilde, dessen histologische Structur zweifellos das Vorliegen des gesuchten Organs erweist. Wenn die Steissdrüse auch keine specifischen Elemente besitzt, so ist doch die Anordnung der sie constituirenden Gefässbildungen charakteristisch genug, um sie auch inmitten der umgebenden Weichtheile sicher zu constatiren: Die Glomeruli sind eingebettet in eine Bindegewebsmasse, welche sich durch ibren Zellieichthum von der kernärmeren Umgebung ziemlich scharf abhebt und einzelne regellos verlaufende Züge glatter Muskelfasern einschliesst. Die Schläuche und Blasen seibst erscheinen im durchschnittenen Zustande nicht anders, als Blutgefässe, nur dass die von einer feinen elastischen Membran und einer Endothellage bekleidete Muskelschicht ebenso wie die dieselbe umgebende bindegewebige Adventitia eine im Verhältniss zum Lumen öbermässige Ausbildung erfahren haben. Auch in den dünn hergestellten Schnitten begegnet man wegen der vielen Windungen und Knickungen häufig von der Fläche gesehenen Abschnitten der Gefässe, welche dann den Eindruck erwecken, als sei ibr Lumen von den Endothelmassen vollständig erfüllt. Die mikroskopischen Bilder, welche ich mir von dem gleichen Organ mebrerer Kinder in den ersten Lebensmonaten darstellte, bestätigten durch ibre vollkommene Uebereinstimmung mit jenen die Richtigkeit meiner Vermuthung.

Die kugligen Gebilde, welche die eigentliche Geschwulstmasse darstellen, erscheinen auf dem Durchsehnitt cystös: Die Hohlräume sind von verschiedener Ausdehnung, stecknadelkopf- bis erbsengross, besitzen eine glatte Innenfäche und beherbergen nur zum kleinen Theil eine bröckelige, käsige, leicht ausdrückbare Substanz; das Gewebe, welches die Cysten einschliesst, ist von grauer, stellenweise rötblicher Farbe, weich, schwammig und locker, und nur an der Peripherie jedes Körpers zu einer etwas festeren Grenzmembran verdichtet.

Im mikroskopischen Bilde kehrt an allen von versehiedenen Stellen des äusseren wie des intraabdominellen Abschnittes der Geschwulst entnommenen Stücken derselbe bistologiscbe Bau wieder: die schon makroskopisch sichtbaren Hoblräume sind mit einem fast durchweg mehrfach geschichteten Epithel ausgekleidet; in einzelnen besteht dasselbe aus exquisit cylindrischen Zellen mit scharfem Saum, um deren verjüngte Basis herum regelmässig angeordnete Ersatzzellen liegen; in den Cysten jedoch, wo die Schicht eine vielfache ist, nehmen die Epithelien polygonale Gestalt an, und ihre innere Grenze wird ungleichmässiger. Ein deutlicher Flimmersaum ist nirgends aufzufinden. In die grösseren Lumina springen papillenartige Fortsätze vor, meist wit einem centralen Bindegewebsstock, bisweilen auch desselben entbehrend als solide Epithelzapfen; ebenso treiben viele der Epithelmembranen 
nach aussen zu knospenförmige Fortsätze. Das Stroma, in welchem diese Hohlräume liegen, stellt ein sehr kernreiches junges Bindegewebe mit spärlicher Intercellularsubstanz dar, dessen Zellen regellos angeordnet erscheinen; nur in der Umgebung einzelner Cysten nimmt es, indem zugleich seine Elemente platt und länglich werden, eine concentrische Schichtung an und bildet so eine Art Adventitia um die Epithelschläuche. Dieses an einigen Stellen gallertig degenerirte Bindegewebe beherbergt eine ziemlich bęträchtliche Zabl von meist kugligen kleinen Heerden hyalinen Knorpels; und zwar findet sich derselbe in allen Stadien seiner Entwickelung vor, in der durch grossen Gebalt an Zellen bei sehr geringer Zwischensubstanz charakterisirten Jugendform und von dieser an in fortschreitender Ausbildung bis zum ansgeprägten hyalinen Typus. Die meisten dieser Heerde sind durch ein aus platten, concentrisch geschichteten Zellen gebildetes Pericbondrium umschlossen. Endlich finden sich wehr oder weniger lange und breite Züge glatter Muskelfasern in das Grundgewehe eingebettet, deren einzelne wohl die Cysten umschliessen, während die meisten eine gesetzmässige Anordnung vermissen lassen. Auffallend gering ist an allen Stellen der Geschwulst die Zahl der Blutgefässe.

Dieser histologische Befund berechtigt dazu, die vorliegende Neubildung den Cystosarcomen früherer Autoren gleichzustellen, welche nach v. Bergmann ${ }^{1}$ ) nicht, wie angenommen wurde, eine Einlagerung von epithelbekleideten Hohlräumen in ein Grundgewebe von gleichmässigem und einfach "sarcomatösem" Typus darstellten, sondern eine Combination verschiedener Formen der Bindesubstanzgruppe mit eingelagerten Epithelschläuchen, durch deren Erweiterung der cystische Charakter sich ausbildet.

Die Literatur über Sacraltumoren, welche sich zum grössten Theil aus Einzelmittheilungen zusammensetzt, vollständig zu übersehen, ist nicht leicht, und es mögen mir vielleicht manche der bezïglichen Arbeiten entgangen sein. Unter den mir bekannt gewordenen Beschreibungen findet sich keine, welche von einem Nachweis der Steissdrüse neben dem Tumor berichtet. Ich zweifle nicht, dass, wo überhaupt der Versuch gemacht wurde, die Schwierigkeit der Präparation dieses äusserst kleinen Gebildes die Schuld an dem Misslingen trug. Dass gerade im vorliegenden Falle die Auffindung gelang, erscheint deshalb wichtig, weil die Geschwulst alle die Eigenschaften in topographischer und histologischer Beziehung darbietet, welche nach

1) v. Bergmann, Zur Diagnose der angebornen Sacralgeschwülste. Berl. klin. Wochenschr. XXI. 1884. 
Braune's Ansicht zu einer Herleitung von der Steissdrüse berechtigen. Was Braune als charakteristische Kennzeichen anfiuhrt, sind die Lage vor dem Krenz- und Steissbein, ein von Verbiegung dieser Knochen begleitetes Wachsthum nach abwärts unter Einhaltung der Glutäalränder als obere Grenze, eventuell eine zapfenförmige Fortsetzung über den M. levator ani hinaus in die Becken- und Bauchhöhle, die Erweiterung der Art. sacralis media und endlich der cystosarcomatöse Bau.

Wenn die gegen Luschka's Lehre ausgesprochenen Gründe überhaupt noch einer Bestätigung bedurften, so scheint mir der mitgetheilte Fall als solche gelten zu könuen.

Mit der Ausschaltung der Steissdrüse aus der Reihe der Bildungsorgane für die typischen Steissbeingeschwülste soll derselben die Fähigkeit nicht völlig abgesprochen werden, überhaupt ein Neoplasma zu produciren. Wie in jedem mit Perithel bekleideten Gefässbezirk, kann auch in ihr durch Wucherung dieser Elemente ein Angiosarcom sich ausbilden: Buzzi ${ }^{1}$ ) beschreibt ein solches, welches zwischen Kreuzbein and Iastdarm in die Beckenhöhle drang und ausserdem bei Defect des Steissbeins und der zwei unteren Sacralwirbel einen zapfenförmigen Fortsatz in den Sacralkanal schickte; Buzzi selbst gedeukt der Möglichkeit, dass die Steissdrüse den Ausgangspunkt dieser Geschwulst durch Wucherung ihrer endothelialen Bestandtheile darstelle. Braune ${ }^{2}$ ) erwähnt, dass er einmal ein vor dem Kreuzbein liegendes Cylindroma beobachtet zu haben glaubt, ein Gebilde, das heute zu den Angiosarcomen gezählt wird. Möglich ist es, dass auch, wie Buzzi vermuthet, die als Carcinome bei Lotabeck ${ }^{3}$ ) und Braune aufgeführten Fälle Angiosarcome waren, welche ja auch jetzt noch wegen ihres häufig dem Carcinom so überaus ähnlichen Banes die Unterscheidung von demselben schwer machen. Für eine andere Geschwulstform noch könnte ein Zusammenhang mit der Steissdrüse angenommen werden: Dem "Ganglion intercaroticum" Luschka's, welches

1) Buzzi, Beitrag zur Kenntniss der angebornen Geschwïlste der Sacrococcygealgegend. Dieses Archiv Bd. CIX. 1887.

2) Braune, a. a. 0. S. 110 .

3) Lotzbeck, Die angebornen Geschwülste der hinteren Kreuzbeingegend. Nünchen 1358 . 
sich durch Arnold's') Untersuchungen als ein Gefässknäuel erwies, der den Glomeruli caudales ausserordentlich nahe steht, erkennt Arnold die Möglichkeit zu, zum Ausgangspunkt der als Haematocele colli beschriebenen cystischen Blutgeschwülste zu werden; vielleicht darf einer Sacralgeschwulst, welche $\mathrm{Cou}$ Ion ${ }^{2}$ ) beschreibt und der Haematocele colli gleichstellt, der analoge Ursprung aus der Steissdrüse vindicirt werden. Ein höheres Maass von Productionskraft für Geschwülste indessen wird man der Steissdrüse kaum zusprechen können.

Der Auf bau der vorliegenden Geschwulst aus Abkömmlingen verschiedener Keimblätter weist auf die Concurrenz mehrerer Organanlagen bei ihrer Bildung hin, deren monogerminaler Ursprung sich in dem Fehlen eines ausgeprägten fötalen Organs ausspricht. Für die Dislocation von Keimen bietet die Steissbeingegend, in welcher die verschiedenartigsten Abschnürungen der drei Blätter erfolgen, die denkbar günstigsten Bedingungen. Soll der Tumor nach der Herkunft seiner einzelnen Bestandtheile analysirt werden, so müssen die die Hohlräume auskleidenden Cylinderepithelien wobl von dem unteren Darmabschnitt, vielleicht dem postanalen Theil desselben, abgeleitet werden; die Entstehung der mesodermalen Bildungen, des Bindegewebes, des Knorpels und der glatten Musculatur lässt sich schon aus der Verlagerung eines Abschnittes des noch nicht differenzirten mittleren Blattes erklären; wenn indessen der erste Anfang der Geschwulst in eine spätere Zeit verlegt wird, in welcher die Sonderung in die verschiedenen Gewebstypen bereits erfolgt war, so erscheint die Zurückführung der knorpeligen und musculösen Elemente auf das Ende der Wirbelsäule und die Muskelwand des Darms als Ausgangsstätten bei der nahen Nachbarschaft dieser Gebilde wohl zulässig.

Für eine derartige Mischgeschwulst ist die Annahme einos abgegrenzten Gewebscomplexes, in dem sie wurzelt, unnöthig; os wird vielmehr von Zufälligkeiten abhängen, an welcher Stelle der Zusammenstoss der transponirten Keime erfolgt, wie ja das Vorkommen der Cystosarcome bald vor, bald hinter dem Steiss-

1) J. Arnold, Veber die Structur des Ganglion interearoticum. Dieses Archiv Bd. 33. 1865.

2) Coulon, Tumeur du siégc. Crazette bebdomadaire. 1861. p. 36 , 
bein darthut. Und wenn auch bisweilen ein Organ diese Sammelstelle der elementaren Keime repräsentirt, so bietet jedenfalls die Steissdrüse für einen solchen Vorgang nicht nur keine Prädisposition, sondern, wie A rnold betont, sogar möglichst ungiustige Bedingungen dar.

Im Anschluss an den besprochenen Fall mag der Bericht über einen ähnlichen, besonders in klinischer Hinsicht interessanten, Platz finden. Ich verdanke denselben der Güte des Herrn Prof. Kehrer, welcher ihn im Juni 1884 operirt hat und mir darüber folgende Notizen zukommen liess:

Der 4 Tage alte Knabe, welcher rechtzeitig und ohne Schwierigkeit in Schädellage geboren wurde und vollständig wohlentwickelt erscheint, trägt in der Steissgegend eine kleinfaustgrosse Geschwulst ron der Form eines mit der Spitze nach abwärts sebenden Herzens. Während dieselbe in ihrem obersten Abschnitte sich ziemlich gleich weit nach beiden Seiten von der Wirbelsäule des Kindes ausdebnt, weicht ihr unterer Pol nach rechts ab, so dass ihre Hauptmasse nach rechts von der Mittellinie liegt. Die Eläche, in welcher sie dem kindlichen Körper aufsitzt, entspricht der.Gegend zwischen Steissbein und After und dem angrenzenden Theile der rechten Hinterbacke. Das Steissbein scheint normal geformt zu sein, liegt der Hinterfläche des Tumors dicht, flach und kaum beweglich auf und sendet nach dieser durch die Haut fühlbare derbe, faserige Stränge. Die von zablreichen Venen durchzogene Hautbekleidung, welche die continuirliche Fortsetzung der kindlichen Bedeckungen bildet, ist intensiv erysipelatös geröthet und auf der Dorsalfläche in der Ausdebnung eines Fünfmarkstückes ulcerirt. Bis auf eine auf der hinteren Seite der Geschwulst schräg von oben nach unten und links verlaufende seichte Furche ist die Oberfäche derselben glatt. Die Consistenz erweist sich sebr ungleich: An einzelnen Stellen ist sie weich, bisweilen deutlich fluctuirend, während an anderen, besonders in der Näbe der Spitze, feste, knochenharte Partien durchgefühlt werden. Der ganze T'umor Jässt sich nur wenig verschieben; Druck auf ibn ist nicht schmerzbaft und ruft keinerlei Nervenzufälle hervor. -- Da ein Zusammenbang der Gešcbwulst mit den Rückenmarkshäuten nach ihrer Lage vor dem Steissbein nicht zu erwarten war, so erschien ihre Entfernung nicht nur erlaubt, sondern wegen der drohenden Fortsetzung der phlegmonösen Entzïndung und der Ulceration auf die Umgebung sogar geboten. Es wurde folgendes Operationsverfabren eingeschlagen: Der Hautüberzug der Geschwulst wurde $1-2 \mathrm{~cm}$ über de! Basis derselben durch zwei bogenförmige vom Steissbein nach dem After ziehende Schnitte durchtrennt; danach liess sich tie Auslösung aus der nur durch lockeres Gewebe hergestellten Verbindung mit den Glutäalmuskeln und der Hinterfläche des verlängerten Mastdarms, welcher letzterer in einer Längenausdehnung von circa $5 \mathrm{~cm}$ freigelegt wurde, leicht bewerkstelligen; in- 
dessen waren die vom Steissbein ausgehenden Stränge so fest, dass von ihrer Durchtrennung abgesehen und dieses vom Kreuzbein abgeschnitten und mit dem Tumor entfernt wurde. Einige in das Cavum recto-ischiadicum hineinragende cystische Vorwölbungen wurden bei der Operation eröffnet und liessen einen weissen gallertigen Inbalt ausfliessen. Ueber der handtellergrossen, trichterförmig vertieften Wundfäche wurde unter Einführung eines gebogenen Gummidrains die Baut vereinigt und vor dem Anlegen des Verbandes längs der Nahtlinie Collodium in mehreren Schichten aufgetragen, um die Berührung mit Fäcalien zu verhüten. Die Wundheilung erfolgte per primam intentionem bis auf die zwei Drainstellen, welche sich durch GranuJation schlossen, und am 30. Tage nach der Operation wurde das Kind, nachdem es einen Darmkatarrh überstanden hatte, geheilt und mit durehweg normalen Functionen entlassen. Als ich dasselbe im Januar d. J., also $3 \frac{1}{2}$ Jabre nach der Operation wieder untersuchen konnte, fand ich es in seiner Entwickelung nach jeder Richtung normal fortgeschritten, kräftig geworden und frei von jeder Beschwerde. Anffallend war nur das etwas magere Aussehen der Glutäalgegenden. Durch äussere Palpation und durch Rectaluntersuchung liess sich der Defect des Steissbeins fühlen; in der Mitte der fast sagittal verlaufenden strichförmigen Operationsnarbe lag ein erbsengrosses Narbenknötchen, welches nach Aussage des Vaters bis October vorigen Jabres bisweilen genässt hatte, seitdem aber vollständig geschlossen geblieben war.

Der in Müller'scher Flüssigkeit gebärtete Tumor, welcher sich aus der äberziehenden Haut leicht auslösen lässt, erscheint auf dem Durchschnitt cystös; die Cysten varïren im Unfang von der Grösse eines Stecknadelkopfes bis zu der einer Haselnuss; die grössten liegen in der peripherischen Zone. Ein Theil von ihnen schliesst käsigen, geronnenen Inhalt ein, andere sind leer und zeigen eine glatte, glänzende Innenfläche. Das Gewebe, in welchem diese Hohlräume eingebettet liegen, besitzt zwischen den grösseren derselben hänfig nur die Dicke einer membranösen Scheidewand; an anderen Stellen aber, besonders im centralen Theile der Geschwulst, tritt es in breiten Zägen auf und erscheint hier fibrös, bald ziemlich fest, bald weicher, und schliesst in unregelmässiger Vertheilung äusserst kleine, weisslich glänzende Heerde von Knorpelconsistenz ein.

Die histologische Architectur, wie sie im mikroskopischen Bilde sich zeigt, stimnt ziemlich vollständig mit der des erstbeschriebenen Falles überein: Die Oysten tragen eine meist mehrschichtige Epithelauskleidung und sind zum Theil durch concentrisch angeordnete lange, spindelige Zellen von der Umgebung abgegrenzt. Das Stroma stellt wiederum ein Gemisch mesodermaler Gewebsformationen dar; den Hauptbestandtheil bildet kernreiches, jugendliches Bindegewebe, welches die schon makroskopisch wabrnebmbaren Knorpelinseln einschliesst und von Zügen glatter Musculatur durchsetzt wird. Die Unterschiede gegenüber dem ersten Fall sind fast nur quantitative: Die Cysten treten in geringerer Zabl auf, sind im Allgemeinen grösser und treiben weniger reichliche knospenförmige Auswüchse nach aussen und papilläre Vorsprünge nach innen; ebenso sind die Knorpelheerde spärlicher 
vorhanden, dagegen findet sirh eine bedeutend stärkere Vascularisation; enillich heben sich gegenüber der ziemlich gleichmässigen Vertheilung der Kerne in dem bindegewebigen Bestandtheil in jenem Falle hier vielfach sehr kernreiche Bezirke von kernärmeren $a b$, welche letztere nicht selten fibrilläre Streifung annehmen. Eine Eigenthümlichkeit, welche im ersten Falle fehlt, besteht in dem Auftreten einzelner kleiner, vollständig ausgebildeter acinöser Irüsenbezirke.

Durch die beschriebene Zusammensetzung ist auch dieser Tumor als eine "cystosarcomatöse" Mischgeschwulst charakterisirt.

Die Therapie hat ziemlich selten Gelegenheit gehabt, sich an "Cystosarcomen" zu versuchen, da dieser Geschwulstypus häufiger als alle anderen, einschliesslich der includirten Parasiten, intrauterinen Tod ihres Trägers, oder Absterben desselben während der Geburt, oder Lebensunfähigkeit wegen za früher Geburt veranlasst. Die Erfahrungen, welche an den wenigen zur Operation gekommenen Fällen gemacht wurden, stellen die "Cystosarcome" ebenfalls im Vergleich zu den übrigen Geschwulstformen als die prognostisch ungünstigste dar: Während die Exstirpation von Lipomen fast durchweg, die der cavernösen Lymphangiome meist, und die der Infötationen wenigstens sehr häufig glücklichen Verlauf nahmen, berichtet die Literatur für jene fast immer von tödtlichem Ausgang: Bei Braune findet sich keine operative Entfernung eines "Cystosarcoms" mit günstigem Erfolg verzeichnet. Molck ${ }^{3}$ ) führt in einer späteren Statistik nur 1 Heilung unter 12 operirten Fällen des gleichen Geschwulsttypus auf.

1) Molck, Thèse, Strassbourg 1868, cit. von Lachaud a. a. 0. 